\title{
Screening Mental Well-Being in High Demand Occupational Settings in South Africa
}

\author{
Charles H Van Wijk \\ South Africa
}

doi: 10.19044/esj.2017.v13n14p140 URL:http://dx.doi.org/10.19044/esj.2017.v13n14p140

\begin{abstract}
This study considered promoting well-being of employees in high demand occupational positions, through the screening of mental well-being. The numerous challenges to such monitoring in a multi-lingual developing country like South Africa - where this study was conducted - requires a tool that can screen for the mental well-being of at-risk individuals, in order to timeously refer vulnerable persons to appropriate mental health support services.

This paper explored whether the State-Trait Personality Inventory-Trait Scales (STPI-T) can be used fairly to monitor the well-being of young adult South Africans in high demand occupational settings. The self-report scales were administered to a non-clinical sample (women=392, men=521) aged 20 to 40 years, and their responses were analyzed with MANOVA and a ROC analysis.

The main findings of the study indicated that contextualised norms are required (and were provided for the sample studied), develop per specific national and occupational environment; and that socio-demographic variables like gender, age, and language had limited effect on scale responses in this select sample. The scales effectively identified vulnerable individuals, comparable to psychological interviews. Scale scores were highly homogenized, facilitating identification of outliers which may indicate poorer mental health, thus enabling further referral for appropriate support.

In conclusion, the study provides support for the use of the SPTI-T to promote the well-being of employees in multi-lingual high demand occupational settings, through regular monitoring and early identification of risk for poor mental health.
\end{abstract}

Keywords: Mental well-being; screening; norm data; psychological scales 


\section{Introduction}

The pace of development in modern society is creating an increasing need for high demand occupational posts. These are positions that require employees to be alert, able to make decisions in short timeframes, and take independent action in response to ever changing environmental situations. Examples include personnel working as control center monitors, in emergency services and public security, as well as military personnel working in various technologically demanding environments. Due to the high demand nature of such posts, mental health concerns may have significant consequences, e.g. for the safety of both smaller groups and larger segments of society. Occupational health surveillance has become one mechanism to facilitate safety in high demand occupational contexts.

High demand occupational settings often equates to high stress (Adriaenssens, De Gucht, \& Maes, 2015; Liberman, Best, Metzler, Fagan, Weiss, \& Marmar, 2002). One way to promote the well-being of employees in high-demand occupational settings is through the early identification of risk for poor mental health, which would then allow for timeous referral to appropriate support.

Within the South African (SA) occupational environment, serious challenges exists in the monitoring of mental health and well-being in the workplace. These include the national mental health environment, the national labor market, issues around language and culture, and the limit on available resources.

National mental health environment. SA studies describe a high risk mental health environment. About $16 \%$ of South Africans suffered from a common mental disorder in the previous year (Lundt, Kleintjes, Kakuma, \& Flisher, 2009), and about one in three South Africans will suffer from a psychiatric disorder at some point in their lives (Foxcroft \& Roodt, 2009). Post-traumatic stress disorder (PTSD) continues to be a significant problem for public health in SA, affecting individuals in all sectors of society, with most South Africans experiencing at least one traumatic event in their lives, and the majority reporting multiple events. While not all trauma leads to PTSD, it has been positively related to increased distress (Edwards, 2005; Seedat, Le Roux, \& Stein, 2003; Williams, Williams, Stein, Seedat, Jackson, \& Moomal, 2007). The prevalence of mental health difficulties potentially places a significant number of employees in high-demand positions at risk for poor mental well-being.

National labor market. The high unemployment in SA adds to the psychological burden of employees, through the pressure to remain employed. To ensure this, SA workers spend more time at work compared to other countries (LexisNexis, 2010), and this includes both white and blue collar workers (De Klerk \& Mostert, 2010). This may result in work-related 
stress, burnout, and depression (De Klerk \& Mostert, 2010). Employees may also be the only breadwinner in formal employment and financially responsible for their extended family, which apart from the potential stress associated with that, may also lead to under-reporting of mental health concerns.

Language and culture. SA's rich diversity allows for 11 official languages, with a complex mix of different culture and schooling backgrounds. This creates challenges in measuring and interpreting the expression of poor mental well-being among individuals who have English as a second language. For example, the level of English of established scales are often inappropriate for local use, and educational disparities further raise concerns regarding the validity of the idiomatic English of comprehensive scales which largely originates from the industrialized world.

Limited resources. SA is a developing country with limited resources for occupational health surveillance. In organizations with large numbers of employees to monitor, detailed individual mental health assessment is often impractical. While a variety of psychopathology measures exists for detailed assessment in group formats, such comprehensive psychometric instruments also carry significant costs, which place them out of the reach of most occupational health surveillance programs.

Screening in order to identify-for-referral would be particularly useful in a country with high levels of mental health presentation. Brief screening in group format appears to hold promise, although it does pose challenges in choosing the most appropriate screening tool. Available psychometric screening tools further pose their own challenges, including: (a) inadequate or inappropriate norming; (b) difficulties with language (most have difficult English vocabulary, used with individuals who may not have English as first language); and (c) the lack of resources to procure expensive and sophisticated tests.

For these reasons, few of the traditional comprehensive psychological assessment instruments seem particularly well suited for the purpose of screening well-being in the multi-lingual SA context. What is required is a brief instrument that could appropriately screen for distress, to ensure the safety of both individuals and work-groups in high demand occupational settings.

\section{State-Trait Personality Inventory}

The State-Trait Personality Inventory, Form Y (STPI; Spielberger, 1995) has shown promise within the context of high demand occupational settings. Its trait-anxiety subscale has been used previously with air traffic control specialists (ATCS; Collins, Schroeder, \& Nye, 1989), as well as with subaquatic specialists (Van Wijk, 2014), and good predictive validity was 
reported in both cases. Further, acceptable psychometric properties for a SA student population has been reported (Du Plessis, 2014).

The STPI is a self-administered questionnaire designed to measure transitory and dispositional anxiety, curiosity, anger, and depression in adults (Spielberger \& Reheiser, 2009). The 'state' items aim to assess a subject's current emotional state, while 'trait' items aims to assess the subject's emotional disposition. Each sub-scale has 10 items, with each item rated on a four-point frequency scale. The STPI trait scales (STPI-T) taps into four personality traits (also referred to as emotional dispositions), namely in anxiety (Ax), curiosity (Cy), anger (Ag) and depression (Dp). These traits are associated with vulnerability or resilience to psychiatric conditions, e.g. depression (Hankin, 2010), substance use disorders (Belcher, Volkow, Moeller, \& Ferre, 2014), and post-traumatic stress disorder (Jakšić, Brajković, Ivezić, Topić, \& Jakovljević, 2012). This is also pertinent in the case of high demand occupations, given the potential stressful nature of such work environments.

A detailed history of the development of the STPI (Form Y) can be found in Spielberger and Reheiser (2009), who defined the trait subscales in terms of relatively stable individual differences in $\mathrm{Ax}, \mathrm{Cy}, \mathrm{Ag}$, and $\mathrm{Dp}$ proneness as reflected in the frequency that such states have been manifested in the past and the probability that such emotions will be experienced in the future. People with higher scores on the trait scales would be more prone to experience the emotional states associated with that trait (Spielberger \& Jacobs, 1982). While Ax, Ag, and Dp are indicators of proneness to emotional distress, Cy is a predictor of emotional wellbeing (Spielberger \& Reheiser, 2009), and may provide a measure of resilience to the stressors reviewed earlier.

Spielberger and Reheiser (2009) contend that "manifestations of anxiety, anger, depression and curiosity can be considered critical psychological vital signs that are strongly related to an individual's wellbeing. Variations in the intensity and duration of these emotions provide essential information about a person's mental health that help to identify recent events and longstanding conflicts that have a significant impact on the individual's life” (p.272).

It is proposed here that measuring dispositional emotional traits may thus confer two benefits: Firstly, it might be possible to predict the probability of future mental health concerns by examining the vulnerability factors Ax, Ag and Dp, and the resilience factor Cy. Scores on these scales may predict coping with stressors (or proneness to poor coping), and in doing so assess the risk for subsequent adverse mental health outcomes and need for further intervention. Secondly, trait scores may provide a reflection of current well-being, through its sensitivity to the impact of recent events as 
well as longstanding intrapsychic issues, which can then guide further intervention.

The potential to predict risk (for the purpose of early intervention) may give the STPI-T an advantage over symptom checklists, like the widely used General Health Questionnaire (Goldberg, 1992), which generally tend to focus on current distress.

The STPI Form Y can rapidly and easily be administered through scoring procedures that are objective, yielding results that are unbiased by the test administrator (Spielberger \& Reheiser, 2009). The trait section is brief - only 40 simple sentence items - and appears to employ basic English, which makes it appealing for a multi-lingual population where many do not have English as first language. Available norms are based on USA samples (Spielberger, 1995), which pose the question whether there is a requirement to develop local norms.

\section{STPI-T in the South African context}

SA has a heterogeneous society, and to measure constructs like emotional disposition requires a psychometric tool that is reliable, valid and fair to different groups (Foxcroft \& Roodt, 2009; Laher, 2012). Language is a particular concern, as results obtained from psychological assessments can be significantly influenced should they be written in a language that is not the first language of the test-taker (Van Eeden \& Mantsha, 2007). The STPI items are written in basic English, but it is not clear whether it would be appropriate for a multi-lingual population where English is often the second or third language.

Further, it is equally important to note that language proficiency also remains significantly dependent on the quality of education (Laher \& Cockcroft, 2013). Due to historical as well as contemporary structural challenges which are systemic to SA's education system, post school language proficiency in English is highly variable. Further, different levels of acculturation may compromise the validity of any instrument, and responses to self-report instruments (like the STPI) may be vulnerable to cultural influences (Foxcroft, Paterson, Le Roux, \& Herbst, 2004; Foxcroft \& Roodt, 2009). Culture has been found in previous SA studies to moderate performance and outcomes on psychological measures (Bedell, Van Eeden, \& Van Staden, 1999). At the same time, broad-based cultural tendencies towards collectivism, as in indigenous African cultures, or individualism, as in Western cultures with a European heritage, have also been shown to demonstrate peculiar response styles (Vogt \& Laher, 2009). Collectivist culture have been evidenced to favor less extreme response styles (Allik \& McCrae, 2004), while Euro-American cultures have demonstrated a more extreme response bias (Hamamura, Heine, \& Paulhus, 2008). Against this 
backdrop, the development of local norm bases for internationally normed mental health assessment measures have moved to the forefront of mental health assessment in SA (Laher \& Cockcroft, 2013).

The challenges for language and culture is not only an African phenomenon, and can be found in many diverse settings - for example from the USA to the Middle-East - where language may be shared but cultural expressions vary.

A comprehensive evaluation of the psychometric properties of the STPI (Form Y) using a large SA student sample originating from diverse backgrounds, reported Cronbach alpha reliability coefficients from satisfactory (Cy) to good (Ax, Ag, Dp) for the trait scales, as well as generally good construct validity (Du Plessis, 2014). There was little evidence of differential item functions for gender or race, and it was concluded that individual item difference was unlikely to result in bias at scale level (Du Plessis, 2014). The students in the sample further indicated terms that were unknown or ambiguous, and between $1.6 \%$ and $6.6 \%$ reported difficulty with three items (no 44,54,63). Removal of problem items did not impact on scale reliability in any significant way (Du Plessis, 2014).

\section{Rationale and aims}

There is a need for an instrument to screen for mental well-being within the context of high demand occupational settings. The STPI-T appears to offer an opportunity to firstly monitor current well-being/distress of employees in non-clinical, high demand occupational settings. It would do this through its sensitivity to the impact of recent events as well as to longstanding intrapsychic issues. Given the high prevalence of anxiety and depression in general SA society, with limited mental health resources available, such monitoring would enable timeous referral towards appropriate mental health services. Secondly, and at least of equal importance, the ability to screen for vulnerability and resilience factors also offer the opportunity to predict coping and the risk for adverse mental health outcomes.

Before widespread use of the STPI-T can be considered, evidence of its usefulness in the local context is required. While its psychometric properties when used with students have been established, university students may have different language skills and possibly different levels of acculturation (Naidoo \& Mahabeer, 2006) than both the general SA population, and employees in specific high demand occupational settings.

This paper reports on an exploratory study which aimed to determine whether the STPI-T can be used fairly to monitor the well-being of employees in high-demand occupational situations, among young adults 
South Africans, in a sample of SA Navy (SAN) sailors aged 20 to 40 years. It set three specific objectives, namely:

1. To determine whether norms from the Manual (Spielberger, 1995), or even student populations, can be used for local screening of high demand occupational personnel, given the SA specific situation reviewed earlier. This will also include scale properties;

2. To determine which socio-demographic variables could be used to inform the reporting format of local norms, if the original American norms cannot be used. These variables include gender, age, and language;

3. To determine whether the STPI-T would be useful in identifying atrisk individuals, in this population of working young adults.

\section{Methods}

\section{Participants}

The sample consisted of 913 SAN volunteers who completed the STPI-T during their annual occupational health screening (see Table 1 for distribution). Participants were included if they were in possession of a National Senior Certificate (68 were also in possession of National Diplomas, and 15 had bachelor degrees), were in the age group 20 to 40 years, and had no acute psychiatric conditions at the time. Language distribution was representative of the SAN, while women were overrecruited to enable gender comparisons.

The age and qualification criteria were chosen for (a) comparison with both the original normative sample and the existing SA student sample, and (b) the general requirements of high-demand occupations.

Age, gender, and language were recorded for each participant. The group was considered heterogeneous, diverse in acculturation and language and quality of education.

Table 1: Distribution of participants in terms of age, gender, and language

\section{Gender}

\begin{tabular}{ccc} 
Age & Female & Male \\
\hline $18-22$ & 40 & 81 \\
$23-32$ & 287 & 285 \\
$33-40$ & 65 & 155 \\
& $43 \%$ & $57 \%$ \\
\hline \multicolumn{3}{c}{ Language } \\
\hline English & 194 & $\%$ \\
isiXhosa & 136 & 21.2 \\
Afrikaans & 132 & 14.9 \\
seTswana & 108 & 11.8 \\
isiZulu & 93 & 10.2 \\
sePedi & 86 & 9.4 \\
\hline
\end{tabular}




\begin{tabular}{ccc}
\hline seSotho & 84 & 9.2 \\
tshiVenda & 20 & 2.2 \\
tshiTsonga & 16 & 1.8 \\
siSwati & 14 & 1.5 \\
Ndebele & 9 & 1.0 \\
Unknown/other & 21 & 2.3 \\
\hline
\end{tabular}

\section{Measures}

The STPI-T was administered in its standard format. A detailed history of its development can be found in Spielberger and Reheiser (2009). The research manual reported excellent internal reliability, and presented the original normative data for female and male groups separately, and per age categories 18-22, 23-32, and 33+ (Spielberger, 1995). The rationale for this specific grouping was not reported.

Respondents also participated in a brief interview to assess their level of emotional well-being or distress (to identify at-risk employees), and were allocated a binary category of either good or poor emotional adjustment. Interviews took place on the same day as STPI-T administration, and were conducted by psychologists who were naïve to the STPI-T scores. In total, $7.1 \%(\mathrm{~N}=65)$ of the sample was identified with elevated emotional distress.

\section{Ethics}

The study was conducted according the principles set out in the Declaration of Helsinki (2013). All participants were volunteers and gave informed consent that their data may be used for this analysis. They were informed that no clinical decisions during their occupational health assessments would be made on the basis of STPI-T scores, and that all data would be entered into the database anonymously, to maintain its confidentiality.

\section{Data-analysis}

Statistical analysis of the data was done electronically, using SPSS version 23. Scale scores were compared to the original norms as described in the Manual (Spielberger, 1995), and to SA student data (Du Plessis, 2014), using t-tests for single samples.

Socio-demographic effects were investigated as follow: (a) gender effects across the four scales were examined using MANOVA. Although this was not important among university students (Du Plessis, 2014), it would be important when determining whether separate gender norms would be required, as was reported in the Manual (Spielberger, 1995); (b) the fair use of the scale across language groups was examined by collapsing the 10 African languages into one group for comparison with English, across the four scales, using MANOVA. A sub-sample $(\mathrm{N}=393)$ were requested to indicate problematic items; (c) age effects were examined using Pearson's $r$, 
as well as MANOVA. This was to determine whether separate age group norms would be required, as was reported in the Manual (Spielberger, 1995), and if so, which age categories to use.

The usefulness of the four trait scores to differentiate between good and poorer emotional adjustment in this non-clinical sample was explored using MANOVA. Further, a ROC analyses, as well as sensitivity and specificity calculations, were done to examine the usefulness of the STPI-T to predict emotional distress.

Additional scale characteristics - referring to internal consistency (Cronbach alpha coefficients) - are also reported. Finally, reference data for young adult employed South Africans are presented in tabular format.

\section{Results}

\section{Objective 1: Comparison to existing normative data}

The study sample reported scores that were significantly different from both the original USA norm sample, and the SA student sample (Table 2), on all four scales, in all the gender-by-age categories. The SAN sailors consistently scored in the direction of lesser distress, with moderate to large effect sizes.

Table 2: Outcome of single sample t-tests comparison with available reference groups

\begin{tabular}{|c|c|c|c|c|c|c|c|}
\hline Scales & $\begin{array}{l}\text { Reference } \\
\text { group }\end{array}$ & mean & SD & $t$ & $p$ & M diff & Cohen's d \\
\hline \multicolumn{8}{|c|}{ 18-22 years } \\
\hline \multicolumn{8}{|c|}{ Women $(\mathrm{N}=40)$} \\
\hline $\mathrm{Ax}$ & Original USA & 16.53 & 4.7 & -3.872 & $<.001$ & 2.9 & 0.57 \\
\hline Cy & norm sample & 29.30 & 3.6 & 2.984 & $<.01$ & 1.7 & 0.38 \\
\hline $\mathrm{Ag}$ & from Manual; & 17.68 & 5.0 & -3.172 & $<.01$ & 2.5 & 0.49 \\
\hline \multicolumn{8}{|c|}{ Men $(\mathrm{N}=81)$} \\
\hline$A x$ & 1995, Table 12 & 16.44 & 3.6 & -6.704 & $<.001$ & 2.7 & 0.64 \\
\hline Сy & & 29.98 & 4.2 & 7.009 & $<.001$ & 3.3 & 0.60 \\
\hline $\mathrm{Ag}$ & & 18.07 & 4.7 & -4.359 & $<.001$ & 2.3 & 0.46 \\
\hline \multicolumn{8}{|c|}{ 23-32 years } \\
\hline \multicolumn{8}{|c|}{ Women $(\mathrm{N}=287)$} \\
\hline$A x$ & Original USA & 14.63 & 3.2 & -17.552 & $<.001$ & 3.4 & 0.80 \\
\hline Сy & norm sample & 29.49 & 4.0 & 2.099 & $<.05$ & 0.5 & 0.10 \\
\hline Ag & from Manual; & 16.40 & 3.9 & -8.845 & $<.001$ & 2.0 & 0.49 \\
\hline \multicolumn{8}{|c|}{ Men $(\mathrm{N}=285)$} \\
\hline$A x$ & 1995, Table 12 & 14.88 & 3.5 & -15.272 & $<.001$ & 3.2 & 0.76 \\
\hline Сy & & 29.51 & 4.2 & 4.110 & $<.001$ & 1.0 & 0.22 \\
\hline $\mathrm{Ag}$ & & 16.19 & 4.4 & -8.852 & $<.001$ & 2.3 & 0.49 \\
\hline \multicolumn{8}{|c|}{ Combined age $^{\mathrm{a}}$} \\
\hline & Original USA & Women & $T=327$ & & & & \\
\hline \multirow[t]{2}{*}{ Dp } & norm sample & 14.22 & 3.4 & -23.055 & $<.001$ & 4.3 & 0.89 \\
\hline & from Manual; & \multicolumn{2}{|c|}{ Men (N=367) } & & & & \\
\hline Dp & $\begin{array}{c}\text { Spielberger, } \\
\text { 1995, Table } 16\end{array}$ & 14.53 & 3.3 & -17.670 & $<.001$ & 3.0 & 0.58 \\
\hline
\end{tabular}




\begin{tabular}{|c|c|c|c|c|c|c|c|}
\hline \multicolumn{8}{|c|}{ Total sample $(\mathrm{N}=913)$} \\
\hline$A x$ & SA student & 14.58 & 3.6 & -48.474 & $<.001$ & 5.7 & 1.26 \\
\hline Сy & sample; Du & 29.95 & 4.2 & 21.465 & $<.001$ & 3.0 & 0.66 \\
\hline $\mathrm{Ag}$ & Plessis, 2014, & 16.19 & 4.3 & -38.037 & $<.001$ & 5.4 & 1.06 \\
\hline Dp & Table 4.1 & 14.05 & 3.3 & -38.333 & $<.001$ & 4.2 & 0.93 \\
\hline
\end{tabular}

Internal reliability. The trait anger scale showed good internal consistency (Cronbach alpha=.80). Trait anxiety and trait curiosity (Cronbach alpha $=.68$ for each) were 'borderline' satisfactory only. In each of these scales withdrawal of individual items did not improve alpha coefficients. Trait depression (Cronbach alpha=.68) were also only borderline satisfactory. Withdrawal of item 44 improved the Dp alpha coefficient to .72.

\section{Objective 2: Socio-demographic effects}

Age. Significant but small correlations were found between STPI-T scores and age, with advancing age associated with lesser distress (Table 3). To determine whether separate age groups would be advantageous when reporting normative data, a MANOVA was conducted (see Table 4). Although a significant statistic was found, post hoc analysis (Tukey) revealed inconsistent differences across the individual year groups. Observation of mean plots (not presented) indicated that reporting results for a single age group when compiling reference data would best reflect current responses.

Table 3: Correlations between age and the four trait scales

\begin{tabular}{ccc}
\hline Scale & $\boldsymbol{r}$ & $\boldsymbol{p}$ \\
\hline $\mathrm{Ax}$ & -.33 & $<.001$ \\
$\mathrm{Cy}$ & .15 & $<.001$ \\
$\mathrm{Ag}$ & -.27 & $<.001$ \\
$\mathrm{Dp}$ & -.22 & $<.001$ \\
\hline
\end{tabular}

Gender. There was no significant effect of gender status on mean scores (Table 4). Further analysis treated the sample as one group.

Table 4: Gender, language, and at-risk status effects on four trait scales, using MANOVA

\begin{tabular}{ccccc}
\hline Variables & F-value $^{\mathrm{a}}$ & $\boldsymbol{p}$ & Wilks $\boldsymbol{\Lambda}$ & $\boldsymbol{\eta}_{\mathbf{p}}^{2}$ \\
\hline Age & 2.813 & $<.01$ & .783 & .059 \\
Gender & 0.997 & .408 & .994 & .006 \\
Language & 2.429 & .046 & .989 & .011 \\
At-risk status & 60.783 & $<.001$ & .789 & .211 \\
\hline \multicolumn{5}{c}{}
\end{tabular}

Language. When language groups were collapsed into two categories - English first language speakers, and non-English first language speakers - a statistical significant difference in mean scores based on 
language emerged (Table 4). The mean scores difference between English first language and non-English first language speakers were statistically significant for Cy only ( $<<.05$, with Bonferroni adjustment). However, due to the small effect size, separate norms for English first language speakers and non-English first language speakers did not seem indicated at this time.

A random subsample $(\mathrm{N}=393)$ were also asked to indicate problem items (i.e. items they were not sure whether they understood correctly). Six items were most often reported as problematic (Items 44,47,50,54,63,66; by between 3.1 and $7.6 \%$ of the sample).

\section{Objective 3: usefulness to identify at-risk individuals}

To determine whether the $7.1 \%$ of the sample classified as potentially at-risk (women $=5.9 \%$, men $=8.1 \%$ ) scored significantly different from the rest across the four scales, a MANOVA was conducted. There were statistical significant differences in mean scores based on at-risk classification (Table 4). The mean score differences were statistically significant for all four scales ( $\mathrm{p}<.001$ with Bonferroni adjustment for each). The large mean differences found on Ax (5.9), Cy (3.0), Ag (5.2), and Dp (5.1), could make this practically useful.

To further explore the ability of the STPI to indicate observable emotional distress, a ROC analysis was conducted. Acceptable AUC for Ax (.85), Ag (.77), and Dp (.85) were found, with somewhat poorer AUC for Cy (.69). The ROC curve is graphically represented in Figure 1.

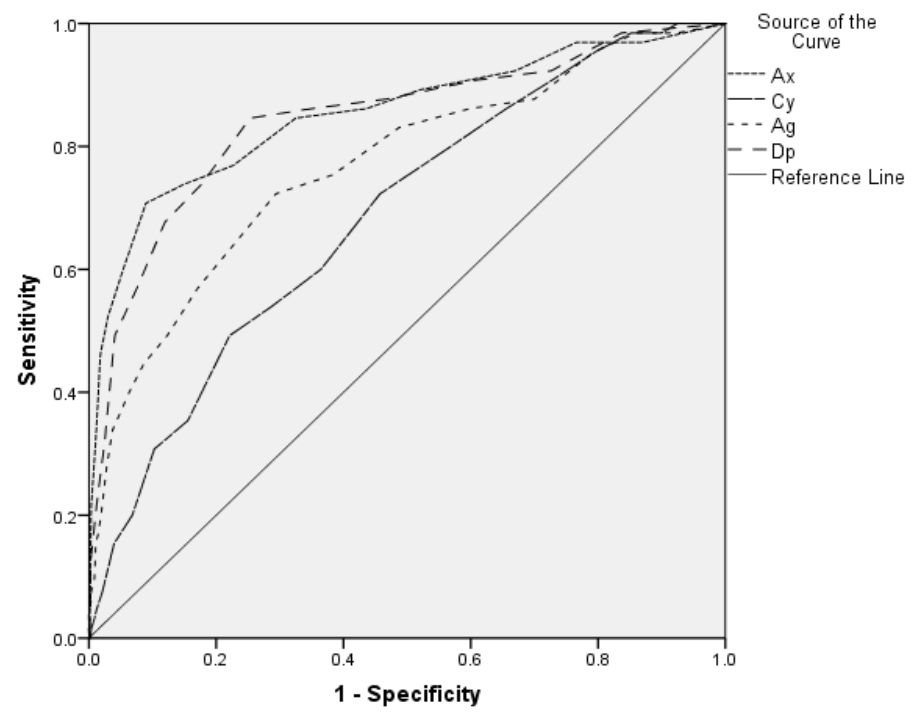

Figure 1: ROC Curve based on interviewer identified at-risk individuals 
Standard deviations are often used to screen for indications of potential distress. The sensitivity and specificity of possible cut-points for STPI-T scores suggesting referral for further assessment are presented in Table 5.

Table 5: Sensitivity and specificity using standard deviation for four dispositional traits

\begin{tabular}{|c|c|c|c|c|c|c|c|c|c|c|c|c|}
\hline \multirow[b]{2}{*}{ SD } & \multicolumn{3}{|c|}{ Anxiety } & \multicolumn{3}{|c|}{ Curiosity } & \multicolumn{3}{|c|}{ Anger } & \multicolumn{3}{|c|}{ Depression } \\
\hline & $\begin{array}{l}\text { Scale } \\
\text { point }\end{array}$ & Sens $^{\mathrm{a}}$ & $\mathrm{Spec}^{\mathrm{b}}$ & $\begin{array}{l}\text { Scale } \\
\text { point }\end{array}$ & Sens & Spec & $\begin{array}{l}\text { Scale } \\
\text { point }\end{array}$ & Sens & Spec & $\begin{array}{l}\text { Scale } \\
\text { point }\end{array}$ & Sens & Spec \\
\hline 0.5 & 16.3 & 78.5 & 78.4 & 27.5 & 53.8 & 71.4 & 18.3 & 64.6 & 78.6 & 15.6 & 84.6 & 76.7 \\
\hline 1.0 & 18.0 & 73.8 & 85.8 & 25.4 & 35.4 & 84.5 & 20.4 & 50.8 & 88.2 & 17.3 & 66.2 & 89.0 \\
\hline 1.5 & 19.8 & 56.9 & 94.7 & 23.2 & 20.0 & 93.2 & 22.6 & 40.0 & 93.7 & 18.9 & 55.4 & 93.1 \\
\hline 2.0 & 21.5 & 50.8 & 98.4 & 21.1 & 7.7 & 97.8 & 24.7 & 27.7 & 96.8 & 20.6 & 30.8 & 98.2 \\
\hline
\end{tabular}

\section{Reference data}

Available statistical exploration of the SAN sample indicated that there was little meaningful effect of gender, age, and language on mean scale scores. It therefore appears at this time that there is no requirement for separate norms, based on currently available socio-demographic variable data. Normative reference data is thus reported for the total group of young adult employed South Africans ( $\mathrm{N}=913)$. Descriptive statistics can be found in Table 6, while Table 7 presents percentiles for the sample.

Table 6: Reference data for young South African working adults (20-40 years; N=913)

\begin{tabular}{ccc}
\hline & mean & SD \\
\hline Anxiety & 14.58 & 3.6 \\
Curiosity & 29.95 & 4.2 \\
Anger & 16.19 & 4.3 \\
Depression & 14.05 & 3.3 \\
\hline
\end{tabular}

Table 7: Percentiles for four emotional traits, both gender, aged 20-40 (N=913)

\begin{tabular}{ccccc}
\hline Percentile & Anxiety & Curiosity & Anger & Depression \\
\hline 95 & 21 & 23 & 25 & 20 \\
90 & 19 & 24 & 22 & 19 \\
75 & 17 & 27 & 18 & 16 \\
50 & 14 & 30 & 15 & 13 \\
25 & 12 & 33 & 13 & 11 \\
10 & 10 & 35 & 11 & 10 \\
5 & 10 & 37 & 10 & 10 \\
\hline
\end{tabular}

\section{Discussion}

It was noteworthy that the SAN sample consistently scored in the direction of better well-being when compared to other groups. Further, scores were relatively homogenized, with low standard deviations reported. A number of factors could be hypothesized to contribute to the homogenization of scores: (a) All participants were SAN sailors, and 
socialization into the organizational culture could be reflected in the scores, in the sense that this is how they would respond to any scale or questionnaire; and (b) the sample were in themselves already highly homogenized, in that all the sailors would have similar entry qualifications, employment status, language exposure, and similar access to organizational welfare support.

A number of factors could also be hypothesized to contribute to the apparent good mental health status of this sample, when compared to other reference groups. Many of these factors are related to the healthy worker effect (HWE; McMichael, 1976; McMichael, Spirtas, \& Kupper, 1974), which suggests that employed people have better health than the general population. One aspect of the HWE has to do with three benefits of the effect of work: Improved access to healthcare, routine disease screening, and physical exercise (Shah, 2009). All of this is present in the current SAN sample. Further, (a) all participants were employed, and in spite of the stress related to employment, could still be considered less stressful than being unemployed; (b) there is good (mental) healthcare available in the SAN; and (c) all participants worked within a group of healthy people, who are required to maintain a certain physical fitness and mental well-being for continuing employment.

Further, data was collected during their annual occupational health screening, which may have introduced a response bias towards reporting better health, in terms of needing to be seen as 'fit for duty', given the potential perceived implications for employment if not.

The lack of significant gender effect was similar to finding from SA students (Du Plessis, 2014), and ATCS (Collins et al., 1989). Scores for Ax, Ag, and Dp decreased, and increased for $\mathrm{Cy}$, with age, but without a clean linear curve of significant differences between subsequent years. This may be partially due to the relatively small age range, and future studies could use a wider range to examine age effects. Language differences, given the small effect sizes, probably had little meaningful effect on scores. No separate norms based on gender, age, or English first language status are indicated at this time. In conclusion, the current SAN population between 20 and 40 only require one normative guide. Although this is different from how STPI results are often reported, there appears to be no compelling evidence to suggest otherwise in this specific occupational context.

In this regard, the study highlighted the need for local norms. The difference from other reference data (both USA and SA) may be a function of both the national (e.g. cultural, language) and the occupational milieu, in particular high demand occupational contexts. For example, a large sample of ATCS trainees at the FAA academy reported similar STPI-T anxiety scores as the SAN sample (women $=14.95$, men $=14.75$; Collins et al, 
1989). In contrast, the SAN sample Ax scores differed from SA subaquatic specialists - another select high demand occupational group (women $=13.29$, men = 12.66; Van Wijk, 2014). Studies comparing anxiety among college students across multiple nationalities found significant differences between the samples from various countries (Baloğlu, Abbassi, \& Masten, 2007). It leads to the conclusion that localized or contextualized norms may be required when conducting mental health screening in specific populations, rather than relying on norms produced from unrelated samples. This may be particularly important for the screening of employees in high demand occupational positions, especially in occupational populations with above average clinical health, where smaller score deviations may indicate mental health difficulties.

The characteristics of the current sample may be shared by other high-demand occupational groups, which allows for some generalization of the findings - for example the homogeneity of responses, and the requirement for localized norms.

Reported scores differentiated significantly between distressed and non-distressed participants in this non-clinical sample. This suggests a high degree of consistent disclosure by the participants: those found to be distressed during the interview also disclosed this in their STPI-T responses. The respondents' consistency - at least in this sample - may go some way to dispel concerns regarding the high face validity of the instrument.

The purpose of employing the STPI is the screening of vulnerability and resilience factors, and the monitoring of current well-being (or distress) of individuals. With local norms now available, it can be used with some confidence to screen individuals in/for high demand occupations in the SA context. In this regard the use of the local norms is recommended, rather than using the original norms in the manual.

While the findings cannot yet be generalized to other multi-lingual national contexts, it does suggest that instruments like the STPI could be used in multi-lingual populations, with the provision that local norms are used to interpret scores.

\section{Limitations and future directions}

In spite of the advantages of the homogenous nature of the group, it may also provide a limitation. The group had at least 12 years of formal education, were employed, and had good access to healthcare. In this regard the SAN sample does not fully represent SA society at large, and the results cannot unreservedly be used with other local populations with different socio-demographic compositions. Different norms for different groups may still be desirable, as the current data only has localized (e.g. context specific) generalizability. Further studies are encouraged to develop specific 
contextualized norms (related to both national and occupational considerations).

Language in the SA context remains a matter of contention, and studies are required to investigate the impact of using alternative words or phrases for problematic items. This in turn may enhance the accessibility of the scale to all South Africans, and address some of the concerns raised by the current requirement of English language proficiency.

\section{Conclusion}

This study found support for the use of the STPI-T to promote the well-being of employees in multi-lingual high-demand occupational settings, through early identification of risk for poor mental health. It highlighted the need to use reference data appropriate to the specific context (national and occupational), and provisional reference data for young employed SA adults working in high-demand occupational contexts were provided.

The STPI-T appears to be a good identifier of at-risk employees, as evidenced by the high correspondence to interview outcomes. Further, the homogenized nature of the reference scores facilitates identification of outlier scores which may indicate poorer mental health, thus enabling timeous referral towards appropriate assessment and support. This makes it useful as a screening tool for the regular monitoring of employees working in high demand occupational settings.

\section{References:}

1. Adriaenssens, J., De Gucht, V., Maes, S. (2015). Causes and consequences of occupational stress in emergency nurses, a longitudinal study. Journal of Nursing Management, 23(3), 346-58. doi: 10.1111/jonm.12138.

2. Allik, J., \& McCrae, R.R. (2004). Towards a geography of personality traits: Patterns of profiles across 36 cultures. Journal of Cross-Cultural Psychology, 35, 58-61.

3. Baloğlu, M., Abbassi, A., \& Masten, W.G. (2007). Cross-cultural comparison of anxiety among college students. College Student Journal, 41(4), 977-984.

4. Bedell, B., Van Eeden, R., \& Van Staden, F. (1999). Culture as a moderator variable in psychological test performance: Issues and trends in South Africa. South African Journal of Industrial Psychology, 25(3), 1-7.

5. Belcher, A.M., Volkow, N.D., Moeller, F.G., \& Ferré, S. (2014). Personality traits and vulnerability or resilience to substance use disorders. Trends in Cognitive Science, 18(4), 211-217. doi: 10.1016/j.tics.2014.01.010. 
6. Collins, W.E., Schroeder, D.L., \& Nye, L.G. (1989). Relationship of anxiety scores to academy and field training performance of air traffic control specialists. Report DOT/FAA/AM-89-7. Civil Aeromedical Institute, Federal Aviation Administration, Oklahoma City, OK. Retrieved from http://www.faa.gov/data_research/research/med_humanfacs/oamtechr eports/1980s/media/am89-07.pdf on 28 December 2016.

7. De Klerk, M., \& Mostert, K. (2010). Work-home interface: Examining socio-demographic predictors in the South African context. South African Journal of Human Resource Management, $8(1)$.

8. Du Plessis, K.E. (2014). An Evaluation of the Psychometric Properties of the STPI (Form Y) for South African Students. Unpublished Masters Dissertation, University of Pretoria. http://hdl.handle.net/2263/43322

9. Edwards, D. (2005). Post-traumatic stress disorder as a public health concern in South Africa. Journal of Psychology in Africa, 15(2), 125-134.

10. Foxcroft, C.D., Paterson, H., Le Roux, N., \& Herbst, D. (2004). Psychological assessment in South Africa: a needs analysis: the test use patterns and needs of psychological assessment practitioners: a final report. Pretoria: Human Sciences research Council. Retrieved from http://www.hsrc.ac.za/en/research-data/view/1642 on 28 December 2016.

11. Foxcroft, C.D. \& Roodt, G. (2009). Introduction to psychological assessment in the South African context. Cape Town: Oxford University Press Southern Africa.

12. Goldberg, D. (1992). General Health Questionnaire (GHQ-12). Windsor, UK: NFER-Nelson.

13. Hamamura, T., Heine, S.J., \& Paulhus, D.L. (2008). Cultural differences in response styles: The role of dialectical thinking. Personality and Individual Differences, 44, 932-942.

14. Hankin, L. (2010). Personality and Depressive Symptoms: Stress Generation and Cognitive Vulnerabilities to Depression in a Prospective Daily Diary Study. Journal of Social and Clinical Psychology, 29(4), 369-401.

15. Jakšić, N., Brajković, L., Ivezić, E., Topić, R., \& Jakovljević, M. (2012). The role of personality traits in posttraumatic stress disorder. Psychiatria Danubina, 24(3), 256-266.

16. Laher, S. (2012). Bias in psychometric studies in South Africa. Retrieved from http://psychologicalassessmentinsouthafrica.com/2012/09/15/explori 
ng-bias-in-the-south-african-context/ on 28 December 2016.

17. Laher, S., \& Cockcroft, K. (2013). Current and future trends in psychological assessment in South Africa: Challenges and opportunities. In S. Laher, \& K. Cockcroft (Eds.), Psychological assessment in South Africa: Research and applications (pp. 120136). Johannesburg: Wits University Press.

18. LexisNexis 2010 international workplace productivity survey (2010). LexisNexis. Retrieved from http://www.multivu.com/players/English/46619-LexisNexisInternational-Workplace-Productivity-Survey/ on 28 December 2016.

19. Liberman, A.M., Best, S.R., Metzler, T.J., Fagan, J.A., Weiss, D.S., Marmar, C.R. (2002). Routine occupational stress and psychological distress in police. Policing: An International Journal of Police Strategies \& Management, 25(2), 421-441.

20. Lundt, C., Kleintjes, S., Kakuma, R., \& Flisher, A.J. (2009). Public sector mental health systems in South Africa: inter-provincial comparisons and policy implications. Social Psychiatry and Psychiatric Epidemiology, 45(3), 393-404.

21. McMichael, A.J. (1976). Standardized mortality ratios and the "healthy worker effect": Scratching beneath the surface. Journal of Occupational Medicine, 18, 165-168.

22. McMichael, A.J., Spirtas, R., \& Kupper, L.L. (1974). An epidemiologic study of mortality within a cohort of rubber workers, 1964-72. Journal of Occupational Medicine, 16, 458-64.

23. Naidoo, J.C. \& Mahabeer, M. (2006). Acculturation and integration patterns among Indian and African university students in South Africa: Implications for ethno-gender relations in the "Rainbow" Nation. Psychology \& Developing Societies, 18, 115-132. doi: 10.1177/097133360501800107

24. Seedat, S., Le Roux, C, \& Stein, D.J. (2003). Prevalence and characteristics of trauma and post-traumatic stress symptoms in operational members of the SANDF. Military Medicine, 168(1), 7175.

25. Shah, D. (2009). Healthy worker effect phenomenon. Indian Journal of Occupational and Environmental Medicine, 13(2), 77-79.

26. Spielberger, C.D. (1995). State-Trait Personality Inventory Research Manual Sampler Set. Mind Garden Inc.

27. Spielberger, C.D. \& Jacobs, G.A. (1982). Personality and smoking behaviour. Journal of Personality Assessment, 46(4), 396-403.

28. Spielberger, C.D. \& Reheiser, E.C. (2009). Assessment of Emotions: Anxiety, Anger, Depression, and Curiosity. Applied Psychology: 
Health and Well-being, 1(3), 271-302. doi:10.1111/j.17580854.2009.01017.x

29. Van Eeden, R., \& Mantsha, T. (2007). Theoretical and methodological considerations in the translation of the 16PF5 into African language. South African Journal of Psychology, 37(1), 6281.

30. Van Wijk, C.H. (2014). The use of Spielberger's State-Trait Personality Inventory (trait anxiety subscale) with naval subaquatic specialists. International Journal of Occupational Medicine and Environmental Health, 27(6), 959-966. doi: 0.2478/s13382-0140321-5

31. Vogt, L. \& Laher, S. (2009). The relationship between individualism/collectivism and the Five Factor Model of personality: An exploratory study. Psychology in Society, 37, 39-54.

32. Williams, S.L., Williams, D.R., Stein, D.J., Seedat, S., Jackson, P.B., \& Moomal, H. (2007). Multiple traumatic events and psychological distress: The South Africa stress and health study. Journal of Traumatic Stress, 20, 845-855. doi: 10.1002/jts.20252 